Proceedings of the International Symposium on Physics of Materials (ISPMA 14), September 10-15, 2017, Prague

\title{
Numerical Simulations of NiTi Shape Memory Alloy Wire Behaviors in Tension, Compression, and Torsion
}

\author{
P. SEDLÁK ${ }^{a, b, *}$ AND M. Frost ${ }^{a, b}$ \\ ${ }^{a}$ Nuclear Physics Institute of the Czech Academy of Sciences, Řž 130, Rež, CZ-25068, Czech Republic \\ ${ }^{b}$ Institute of Thermomechanics of the Czech Academy of Sciences, Dolejškova 5, Praha, CZ-18200, Czech Republic
}

\begin{abstract}
Numerous constitutive models of shape memory alloys aiming to reliable predictions of their remarkable thermomechanical response are available in the literature. Often, experimentally documented specifics as tensioncompression asymmetry or anisotropy of some physical properties of martensite are not considered, e.g. because it is difficult to determine them experimentally without dedicated instrumentation. Hence, parameterization of the models is limited to data from tensile tests, yet the models are used to predict material response in more general loading modes. This contribution addresses limits and pitfalls of such a modeling shortcut. It is also shown that an accurate prediction of NiTi wire response in torsion can be obtained with parameters derived from a test in tension and compression, which challenges the general comprehension of the influence of the processing texture.
\end{abstract}

DOI: 10.12693/APhysPolA.134.842

PACS/topics: 62.20.fg, 46.15.-x

\section{Introduction}

Shape memory alloys (SMA) are intermetallic materials exhibiting the remarkable response to mechanical and/or thermal loading due to a reversible martensitic phase transformation. Their extraordinary properties such as superelasticity, pseudoplasticity or one-way shape memory effect have already been used in various applications [1]. This has simultaneously triggered the development of constitutive models of polycrystalline SMA allowing finite element predictions of the thermomechanical response of final products [2-5], see also recent reviews $[6,7]$.

The crucial issue of SMA modeling is proper capturing and coupling of several deformation phenomena, primarily the elasticity and the formation and changes of the martensitic structure. The latter one - inelastic deformation - is related to the so-called (mean) transformation strain, which is often a key internal variable of the models. The maximum value of transformation strain in SMA single crystals can be deduced from crystallographic considerations and it is highly orientation-dependent and loading mode-dependent for NiTi alloys.

However, to facilitate fitting of material parameters introduced in models, elastic isotropy of individual phases and/or isotropic and symmetric limits of transformation strain, i.e. the von Mises type of transformation domain, are often assumed. Such an approach may be challenged by several experimental facts: (i) tension-compression asymmetry ${ }^{\dagger}$ originating in the crystallographic nature of martensitic transformation has already been well

*corresponding author; e-mail: psedlak@it.cas.cz

†Under compression, the transformation strain induced by the phase transformation is lower and the absolute value of stress level required to start the forward phase transformation is higher than in experiments in tension. documented in literature [8-10], (ii) strong texture of austenite induced during processing of $\mathrm{NiTi}$ components can lead to considerable material anisotropy [11, 12], (iii) different martensitic microstructures form and develop when martensite is subjected to different loading modes [13]; such microstructures can exhibit different macroscopic properties [14, 15]. Accepting these objections, one can further ask how substantial the simulation error is when these effects are neglected in the constitutive model. Of course, the question is relevant only in the loading scenarios where more complex loading modes (than a simple tension) occur. Elementary examples are torsion or bending (combining tension and compression), which are, however, often employed even in the simplest applications [16].

In this contribution, we investigate this issue via comparison of experimental data with simulations performed with our own developed SMA model [17] which is capable to take the abovementioned phenomena into account. We adopt extensive experimental dataset published in [18] containing both tensile and combined tension-torsion tests at different temperatures on a $\mathrm{NiTi}$ wire and complement it by experiments on a similar material in tension-compression published in [19]. As a byproduct, we discuss the influence of material parameters derived from tension and compression to model predictions in torsion.

\section{Constitutive model}

The core of the rate-independent constitutive model for NiTi SMA used in this work was first formulated in [17]. The thermodynamic state of material is described by two external state variables - total strain, $\varepsilon$, and temperature, $T$, two scalar internal variables - volume fraction of martensite, $\xi$, volume fraction of R-phase, $\eta$, - and one tensorial internal variable - traceless inelastic strain related to transformation strain of martensite, $\varepsilon^{\text {in }}$. The rule of mixtures was employed to determine the Helmholtz free energy 


$$
\begin{aligned}
& f\left(T, \varepsilon, \varepsilon^{\text {in }}, \xi\right)= \\
& \quad \frac{1}{2}\left(\varepsilon-\varepsilon^{\text {in }}\right): \hat{C}\left(\varepsilon^{\text {in }}, \xi, \eta\right):\left(\varepsilon-\varepsilon^{\text {in }}\right)+\Delta s^{A M}\left(T-T_{0}\right) \xi \\
& \quad+\Delta s^{A R}\left(T-R_{s}-\frac{\eta\left(R_{f}-R_{s}\right)}{2(1-\xi)}\right) \eta+Q(T),
\end{aligned}
$$

where $Q(T)$ is a term depending on temperature only. $\Delta s^{A M}$ and $\Delta s^{A R}$ denote (positive) change of entropy related to transition between austenite and martensite and austenite and R-phase, respectively. $T_{0}$ is a material parameter related to the thermodynamic equilibrium between austenite and martensite, whereas $R_{s}$ and $R_{f}$ are temperatures adjusting initiation and termination of transformation between austenite and R-phase at the stress-free state. The elastic stiffness tensor of austenite, $\mathrm{R}$-phase and martensite mixture is computed following the Reuss model for strain decomposition

$\hat{C}\left(\varepsilon^{\text {in }}, \xi, \eta\right)=\left[(1-\xi-\eta) \hat{S}^{A}+\eta \hat{S}^{R}+\xi \hat{S}^{M}\left(\frac{\varepsilon^{\text {in }}}{\xi}\right)\right]^{-1}$,

where $\hat{S}^{i}$ denotes the compliance tensor of an individual phase $i$. It is assumed that both austenite and R-phase are elastically isotropic (full stiffness determined by two independent elastic constants: $K$ - bulk modulus and $E$ - Young's modulus), whereas martensite can exhibit substantial anisotropy depending on its internal state (see the next sections for experimental justification). The algorithm for calculation of $\hat{S}^{M}\left(\frac{\varepsilon^{\text {in }}}{\xi}\right)$ was first proposed in [20]. The input parameters of this algorithm are one isotropic elastic tensor of temperature-induced martensite (again two independent parameters only) and two transversely isotropic elasticity tensors of martensite reoriented by applied uniaxial tension ("tensile martensite") and uniaxial compression ("compressive martensite", five independent parameters each), respectively. The elasticity of martensite is determined by a unique decomposition of $\frac{\varepsilon^{\text {in }}}{\xi}$ tensor into tension-like and compressionlike contributions, which modify the isotropic elasticity of temperature-induced martensite, see [20] for details.

The model incorporates natural constraints on volume fractions of phases

$$
0 \leq \eta \leq 1-\xi \leq 1,
$$

and it assumes that the set of possible transformation strain tensors is restricted to a bounded convex domain defined by the inequality

$$
\left\langle\varepsilon^{\text {in }}\right\rangle \leq \xi \text {. }
$$

The specific form of the (convex) function denoted by angle brackets allows capturing the well-documented tension-compression asymmetry or a possibly transformation strain anisotropy. The particular form used in this work follows:

$$
\left\langle\varepsilon^{\mathrm{in}}\right\rangle \leq \xi=\frac{I_{2}\left(\varepsilon^{\mathrm{in}}\right)}{k} \frac{\cos \left(\frac{1}{3} \arccos \left(1-a\left(I_{3}\left(\varepsilon^{\mathrm{in}}\right)+1\right)\right)\right.}{\cos \left(\frac{1}{3} \arccos (1-2 a)\right)},
$$

where $I_{2}(\hat{x})=\sqrt{\frac{2}{3} \hat{x}: \hat{x}}$ and $I_{3}(\hat{x})=4 \frac{\operatorname{det}(\hat{x})}{\left(I_{2}(\hat{x})\right)^{3}}$ for an arbitrary tensor $\hat{x}$.
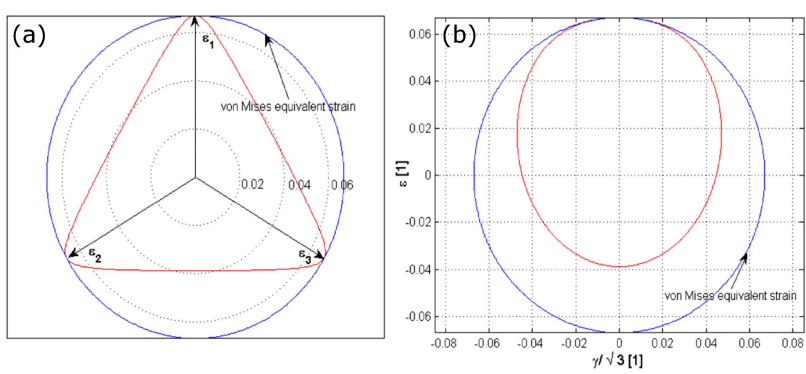

Fig. 1. The shape of the von Mises transformation strain domain (blue) and the domain described by Eq. (5) (red) $(k=0.067, a=0.98)$ in the space of principal strain components (a) - viewed from the (111) direction, and in the plane of tensile $\left(\varepsilon=\varepsilon_{11}\right)$ and shear $\left(\gamma=2 \varepsilon_{13}\right)$ strain components (b).

Here, $k$ is a material parameter representing the maximum transformation strain in tension and the parameter $a$ (ranging between 0 and 1 ) captures experimentally observed tension-compression asymmetry (see the next sections). The forms of the transformation strain domain defined by Eq. (5) for $a=0$ (von Mises domain) and $a=0.98$ are depicted on Fig. 1. The domain is shown both in the space of principal strain components and in the plane of tensile $\left(\varepsilon=\varepsilon_{11}\right)$ and shear $\left(\gamma=2 \varepsilon_{13}\right)$ strain components, which is relevant for simulation of combined tension-torsion experiments. It is worth noting that the definition of the domain by Eq. (5) neglects any possible material anisotropy related to, e.g. crystallographic texture of the material as it is a function only of tensor invariants. Rate-independent dissipation function, which completes the definition of the constitutive model, has the following form (see [21] for more details):

$$
\begin{aligned}
& d\left(T, \varepsilon^{\text {in }} \xi, \dot{\varepsilon}^{\text {in }}, \dot{\xi}\right)=
\end{aligned}
$$

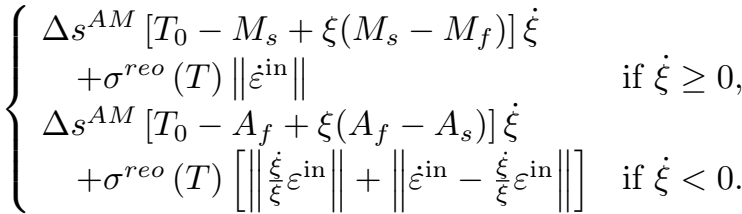

Here, $A_{s}, A_{f}, M_{s}, M_{f}$ and $\sigma^{r e o}(T)$ are material parameters influencing the hysteretic behavior of the model. The dissipation associated with the transition between austenite and R-phase is neglected, hence the dissipation is not a function of $\dot{\eta}$.

\section{Numerical simulations}

Numerical simulations within this work have been performed by our own-developed User MATerial subroutine in numerical software tool Abaqus validated in previous works [22-24]. Based on prescribed strain and temperature, values of internal variables are obtained as the solution of a constrained minimization problem, in which the sum of the free energy and the dissipation function is minimized in each integration step with respect to the internal variables. For more details on numerical treatment and implementation see [17, 21]. Simulations of simple uniaxial tension tests with displacement control 
were done using one 8-node linear brick element, simulations of combined tension-torsion tests were performed using four-node axisymmetric bilinear elements with an additional twist degree of freedom; $500(50 \times 10)$ elements were used to represent a $1 \mathrm{~mm}$ long segment of the wire.

Three complementary sources were used for fitting the material parameters: (a) extended experimental dataset published in [18], (b) superelastic tensile and compressive tests at constant temperature measured in [19], (c) measurements of the elastic anisotropy of strained martensite by ultrasound methods performed in [15].

To demonstrate the effects of tension-compression asymmetry and elastic anisotropy of martensite, we proceed as follows:

1. The model was fitted on "tension at constant temperature" tests and "temperature cycling at constant preloads" tests from [18]. We obtained material parameters listed in Table I except for the bulk moduli of austenite and martensite - these were set to $K^{A}=K^{M}=143 \mathrm{GPa}$, i.e. values determined in $[15]$ - and asymmetry parameter. We set $a=0$ corresponding to isotropic (von Misestype) transformation domain. The Young's modulus of martensite determined from the tensile tests, $E^{M}=43 \mathrm{GPa}$, corresponds well to measurements in [15]. $K^{M}$ and $E^{M}$ were used to set a fixed $\hat{S}^{M}$, hence martensite is supposed isotropic in any loading scenario. This set of parameters - called hereinafter "set 1" - represents the situation when all the material parameters are derived simply from tensile tests as common in modeling with very limited material and experimental sources (e.g. only NiTi wires and a tensile machine with a thermal chamber are available).

2 . In the second set of parameters, "set 2", only the asymmetry parameter was changed: $a=0.98$ (very close to the upper limit) so that the influence of tension-compression asymmetry can be studied. We remind that although the elastic tensors of phases are different, they are all isotropic; such a setting is very common in the most of current NiTi SMA models.
3. Both tension-compression asymmetry $(a=0.98)$ and elastic anisotropy of oriented martensite are included in "set 3". Elastic tensors of strained martensite enter the model via Eq. (2) and the algorithm from [20]. All values of parameters in this set are listed in Tables I and II.

Parameters of the constitutive model (set 3)

TABLE I used in simulations, see text for details.

\begin{tabular}{c|c|c|c}
\hline \hline$K^{A, R}[\mathrm{GPa}]$ & $E^{A}[\mathrm{GPa}]$ & $E^{R}[\mathrm{GPa}]$ & $T_{0}\left[{ }^{\circ} \mathrm{C}\right]$ \\
\hline 150 & 71 & 42 & -27 \\
\hline \hline$M_{s}\left[{ }^{\circ} \mathrm{C}\right]$ & $M_{f}\left[{ }^{\circ} \mathrm{C}\right]$ & $A_{s}\left[{ }^{\circ} \mathrm{C}\right]$ & $A_{f}\left[{ }^{\circ} \mathrm{C}\right]$ \\
\hline-27 & -37 & -22 & -11 \\
\hline \hline$R_{s}\left[{ }^{\circ} \mathrm{C}\right]$ & $R_{f}\left[{ }^{\circ} \mathrm{C}\right]$ & $\Delta s^{A R}\left[\mathrm{MPa} /{ }^{\circ} \mathrm{C}\right]$ & $\Delta s^{A M}\left[\mathrm{MPa} /{ }^{\circ} \mathrm{C}\right]$ \\
\hline 25 & 5 & 0.121 & 0.364 \\
\hline \hline$\sigma_{0}^{\text {reo }}[\mathrm{MPa}]$ & $\Sigma^{r e o}\left[\mathrm{MPa} /{ }^{\circ} \mathrm{C}\right]$ & $k$ & $a$ \\
\hline 110 & -0.8 & 0.067 & 0.98
\end{tabular}

TABLE II

Elastic constants of martensite (A - temperatureinduced martensite, $\mathrm{B}$ - tensile martensite, $\mathrm{C}$ - compressive martensite). The axis of transversal symmetry is aligned with the $x_{3}$-axis.

\begin{tabular}{c|c|c|c|c|c}
\hline \hline & $c_{11}[\mathrm{GPa}]$ & $c_{33}[\mathrm{GPa}]$ & $c_{44}[\mathrm{GPa}]$ & $c_{66}[\mathrm{GPa}]$ & $c_{13}[\mathrm{GPa}]$ \\
\hline $\mathrm{A}$ & 161 & & 15 & & \\
$\mathrm{~B}$ & 179 & 159 & 25 & 32 & 131 \\
$\mathrm{C}$ & 166 & 186 & 30 & 20 & 125
\end{tabular}

\section{Results and discussion}

First, we present results of the fitting procedure leading to set 1. In Figure 2, tensile tests at three different constant temperatures, $-10^{\circ} \mathrm{C}, 10^{\circ} \mathrm{C}$ and $40^{\circ} \mathrm{C}$, are compared with corresponding experiments published in [18]. The correct predictions of the plateau stresses for both loading and unloading shows that the thermomechanical coupling (Clausius-Clapeyron type) is well captured. Let us note that the coupling is sensitive to values of transformation strain and elasticity of all three phases, hence, these parameters are well adjusted. The simulated results are thus equal for all three sets of material parameters described in the previous section.
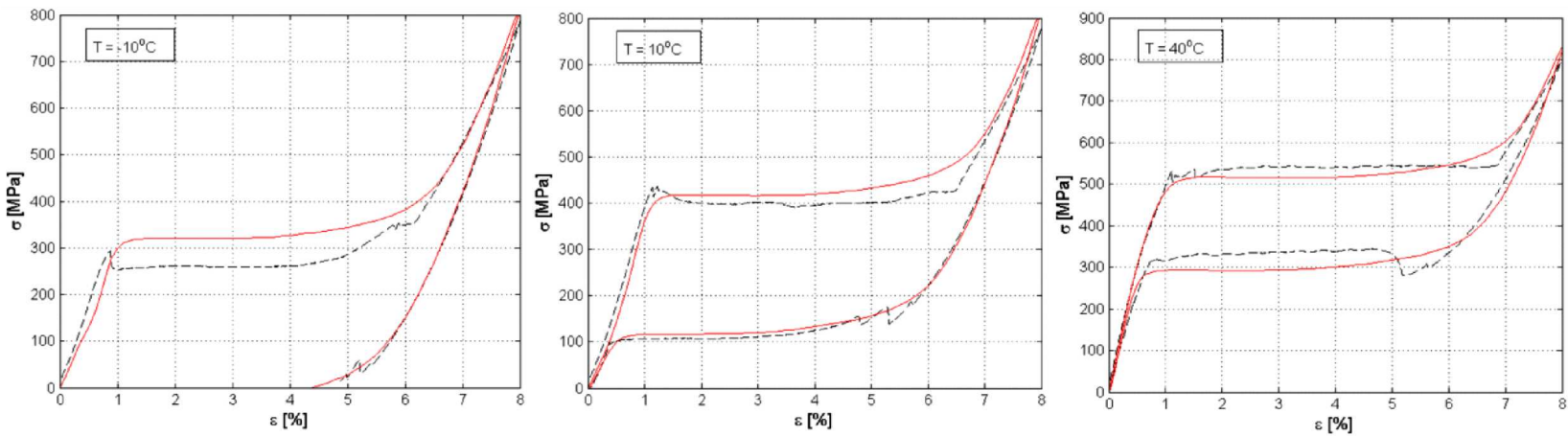

Fig. 2. Simulations of tensile tests (red line) for three different temperatures compared with experiments from [18] (black line). 
Now, we can study the influence of tensioncompression asymmetry and martensite elasticity parameters on the model response. Figure 3a documents very different results obtained for the three sets of material parameters defined in the previous section. By direct comparison with experimental data from [19] in the same figure, we can see large overestimation of transformation strain, if the von Mises-type of transformation domain is assumed. By adjusting the transformation strain via parameter $a$ (i.e. simulation with set 2), the effect of anisotropization of elasticity of martensite induced by reorientation becomes well visible - for a given strain behind the compressive plateau, stress value easily differs by hundreds of MPa. An excellent agreement between the experiment and the simulation is achieved with the set 3 , which reflects both tension-compression asymmetry and martensite anisotropy revealed in experiments. On one hand, this is not so much surprising since martensite elasticity components in set 3 (Table II) were defined so that "best fit" is reached in Figs. 3 and 4 - we hasten to note, however, that they are close to values in [15]. On the other hand, and more importantly, this clearly demonstrates that both phenomena must be taken into account even for a mere uniaxial loading.

Figure $3 \mathrm{~b}$ shows the same analysis of the influence of material parameters on simulations of torque-torsion angle symmetrical $+/$ - responses of a $0.1 \mathrm{~mm}$ thick wire at the constant external tensile stress of $70 \mathrm{MPa}$ and temperature $T=30{ }^{\circ} \mathrm{C}$. The situation is analogous to the compression - a very good fit is obtained by the set 3 of material parameters. Hence, even if no compression is involved in the loading (as in some types of actuators), both discussed material phenomena can severely deteriorate predictive ability of any SMA model which neglects them.
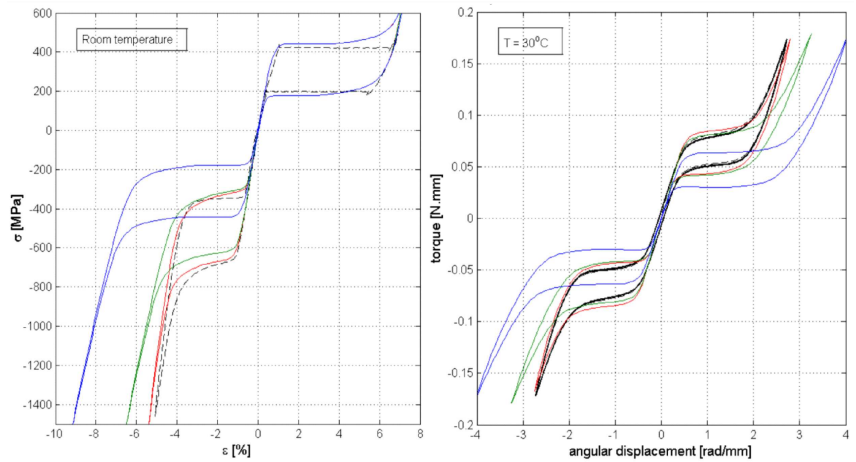

Fig. 3. Simulation of response in tension-compression (left) and torque (right) for three different material parameter sets (see text for details): assuming the von Mises type of transformation surface (blue line), involving tension-compression asymmetry (green line) and considering also the elastic anisotropy of oriented martensite (red line). Experimental data ([19] for tension-compression and [18] for torsion) are marked by black dash lines.
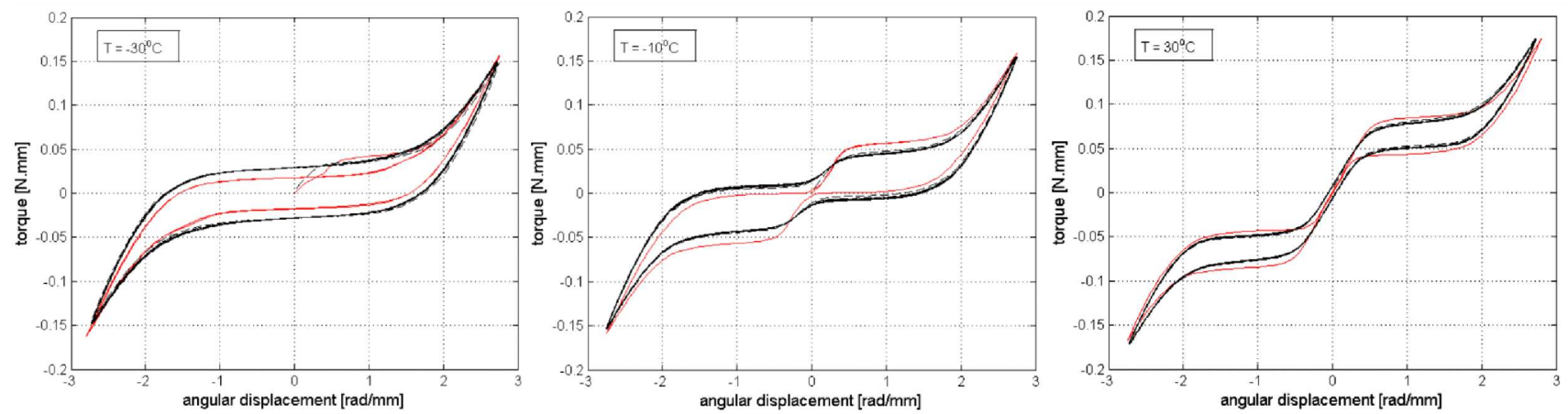

Fig. 4. Simulations of response in torsion tests (red line) for three different temperatures compared with experiments from [18] (black line).

Finally, set 3 was also used to simulate the change of the response in torque with the temperature at the same tensile stress of $70 \mathrm{MPa}$, see Figure 4. All these simulations also show very good agreement with experimental data in many aspects - the transition between pseudoplastic and superelastic behavior, the evolution of plateau stress with temperature, hysteresis width, etc.

One could infer that the good fit is due to an apt parametrization of the transformation domain in Eq. (5) and the advanced algorithm for determination of martensite anisotropy proposed in [20]. However, they both suffer from a major drawback - the initial (e.g. processing) texture of the material is neglected, even though it is most likely present in the wire and should have a substantial impact on its transformation strain and elastic properties.

Indeed, as documented in the literature [13, 25], heat treated drawn NiTi wires exhibit a strong $\langle 111\rangle_{B 2}$ fiber texture along the wire axis in austenite. The simple transition model of [11] shows that the significant anisotropy of martensitic transformation on the level of single crystals gives rise to anisotropy of the transformation domain in polycrystalline SMA (cf. [26]). Hence, the transformation strain in tension in the direction of the wire axis is larger than transformation strains reached in any other direction. 
The question thus arises: How is possible that the value of transformation strain in torsion in the model corresponds to experiments even though the variation of maximum transformation strain in tension and compression with orientation is not taken into account? Within our parametrization (Eq. (5)) and with the maximum compressive strain being lower than the maximum tensile strain, the value of the maximum shear strain is predominantly constrained by the value of the maximum compressive strain. Hence, if the variation of the maximum transformation strain in compression (with orientation) would be much lower than in tension (no experimental data are available to the best authors' knowledge), then the effect would be similar to the transformation strain in shear and the texture-induced anisotropy documented in the literature would have lower impact on the maximum shear strain than generally assumed.

Concerning the elasticity, the influence of texture seems to be moderate according to [15]. In that investigation performed on a textured polycrystalline NiTi sample, low elastic anisotropy was detected in austenite and it even fully vanished during formation of temperatureinduced martensite. After straining in the martensitic state, a relatively strong stiffening was observed in some directions whereas almost no change of the Young's modulus was observed in others, i.e. anisotropy of elasticity indeed developed. However, the stiffening direction mainly followed the orientation of applied loading irrespective of the orientation of the processing texture. In fact, exactly this type of "loading mode-dependent" evolution of elasticity is captured by the algorithm for computation of $\hat{S}^{M}$ in the model.

\section{Conclusion}

This contribution highlights the importance of capturing tension-compression asymmetry and anisotropy of martensite elasticity in simulations of NiTi mechanical response. In contrast to general expectations stemming from the influence of texture, predictions of NiTi wire response in torsion based on material parameters derived from tension and compression tests were found to be fairly accurate. A possible explanation, which must be verified experimentally, was suggested. This finding could have a practical impact as it reduces the number of necessary material parameters and avoids experimental difficulties with testing SMA material in torsion.

\section{Acknowledgments}

This work has been supported by the OP RDE, Ministry of Education, Youth and Sports under the project CZ.02.1.01/0.0/0.0/16_013/0001794 (European Spallation Source - participation of the Czech Republic OP) and by the Czech Science Foundation under the project AdMat, No. 14-36566G.

\section{References}

[1] J. Mohd Jani, M. Leary, A. Subic, M.A. Gibson, Mater. Des. 56, 10781113 (2014).
[2] L.C. Brinson, R. Lammering, Int. J. Solids Struct. 30, 3261 (1993).

[3] J.G. Boyd, D.C. Lagoudas, Int. J. Plast. 12, 805 (1996).

[4] F. Auricchio, R.L. Taylor, Comput. Methods Appl. Mech. Engrg. 143, 175 (1997).

[5] A.C. Souza, E.N. Mamiya, N. Zouain, Eur. J. Mech. A 17, 789 (1998).

[6] C. Cisse, W Zaki, T. Ben Zineb, Int. J. Plast. 76, 244 (2016).

[7] C. Cisse, W Zaki, T. Ben Zineb, Smart Mater. Struct. 25, 103001 (2016).

[8] K. Gall, H. Sehitoglu, Y.I. Chumlyakov, I.V. Kireeva, Acta Mater. 43, 1203 (1999).

[9] L. Orgeas, D. Favier, Acta Mater. 46, 5579 (1999).

[10] S.C. Mao, J.F. Luo, Z. Zhang, M.H. Wu, Y. Liu, X.D. Han, Acta Mater. 58, 3357 (2010).

[11] P. Šittner, V. Novák, Int. J. Plast. 16, 1243 (2000).

[12] Y.C. Shu, K. Bhattacharya, Acta Mater. 46, 5457 (1998).

[13] P. Šittner, P. Lukáš, V. Novák, M.R. Daymond, G.M. Swallowe, Mater. Sci. Eng. A 378, 97 (2004).

[14] P. Šittner, L. Heller, J. Pilch, C. Curfs, T. Alonso, D. Favier, J. Mater. Eng. Perform. 23, 2303 (2014).

[15] M. Thomasová, H. Seiner, P. Sedlák, M. Frost, M. Ševčík, I. Szurman, R. Kocich, J. Drahokoupil, P. Sittner, M. Landa, Acta Mater. 132, 146 (2017).

[16] J. Mohd Jani, M. Leary, A. Subic, J. Intell. Mater. Syst. Struct. 28, 1699 (2016).

[17] P. Sedlák, M. Frost, B. Benešová, T. Ben Zineb, P. Šittner, Int. J. Plast. 39, 132 (2012).

[18] P. Šittner et al., in: ESOMAT 2009 - The 8th European Symposium on Martensitic Transformations, EDP Sciences, paper 08001, 2010.

[19] B.J. Reedlunn, C.B. Churchill, E.E. Nelson, J.A. Shaw, S. Daly, J. Mech. Phys. Solids 63, 509 (2014).

[20] M. Frost, P. Sedlák, L. Kadeřávek, L. Heller, P. Šittner, J. Intell. Mater. Syst. Struct. 27, 1927 (2016).

[21] M. Frost, B. Benešová, P. Sedlák, Math. Mech. Solids 21, 358 (2016).

[22] M. Frost, P. Sedlák, A. Kruisová, M. Landa, J. Mater. Eng. Perform. 23, 2584 (2014).

[23] M. Frost, A. Kruisová, V. Sháněl, P. Sedlák, P. Haušild, M. Kabla, D. Shilo, M. Landa, Acta Phys. Pol. A. 128, 664 (2015).

[24] P. Sedlák, M. Frost, A. Kruisová, P. Šittner, L. Heller, in: Proc. ASME 2014 Conference on Smart Materials, Adaptive Structures and Intelligent Systems SMASIS 2014, ASME, paper SMASIS2014-7465, 2014.

[25] K. Gall, J. Tyber, V. Brice, C.P. Frick, H. J. Maier, N. Morgan, J. Biomed. Mater. Research Part A $\mathbf{7 5}$, 810 (2005).

[26] B. Reedlunn, J.A. Shaw, S. Daly, in: Conference Proceedings from the International Conference on Shape Memory and Superelastic Technologies, San Diego, CA 2017. 